\title{
DESARROLLO MOTRIZ Y ACTIVIDAD FÍSICA EN NIÑOS DE QUINTO DE PRIMARIA DEL TÉCNICO INDUSTRIAL CHIQUINQUIRÁ
}

\section{MOTOR DEVELOPMENT AND PHYSICAL ACTIVITY IN CHILDREN OF FIFTH GRADE OF PRIMARY SCHOOL AT TECHNICAL AND INDUSTRIAL INSTITUTE IN CHIQUINQUIRÁ}

\author{
Fernando Javier Ávila Correa ${ }^{1}$
}

\section{Resumen}

El principal objetivo de la investigación aquí reportada fue el de encontrar la relación entre el desarrollo motriz y la actividad física, en estudiantes de grado quinto del Técnico Industrial Julio Flórez, del municipio de Chiquinquirá, Colombia. Se analizaron los resultados de las nueve pruebas motrices de la batería de test Eurofit y la cuantificación de la actividad física, con el cuestionario QAPACE, en una muestra de 181 escolares ( 75 niñas y 106 niños) con edades entre $9 y$ 12 años. Los resultados mostraron que las mediciones correspondientes a la mayoría de las pruebas físicas presentan diferencias significativas dependiendo del género, excepto para equilibrio y flexibilidad. Los niños mostraron mejores desempeños en las pruebas físicas que las niñas. Frente a la cantidad de actividad física. Los niños registraron valores mayores que las niñas, en ellos se nota una tendencia a la disminución de esta cantidad a medida que aumenta la edad, mientras que en las niñas la cantidad de actividad física es ligeramente constante en términos del aumento de la edad. Finalmente, al realizar la correlación de Pearson a un nivel de significancia del $5 \%$ se encontró que para la mayoría de variables motrices los coeficientes son positivos, aunque pequeños: equilibrio $r=0.025$, fuerza explosiva $r=0.063$, fuerza del tronco $r=0.086$, fuerza isométrica $r=0.087 \mathrm{y}$ resistencia cardio respiratoria $r=0.013$. Los índices reflejan una relación directa pero débil, evidenciando que los niños que practican mayores cantidades de actividad física muestran, ligeramente, mejores condiciones motrices en estos aspectos.

Palabras clave: Propuesta didáctica, aprendizaje colaborativo, enseñanza de circuitos eléctricos.

\section{Abstract}

The general objective of this investigation pretends to find the relationship between motor development and physical activity in fifth grade students at the Industrial and Technical Institute Julio Florez, in Chiquinquirá Colombia.

This study analyzed the results of nine driving tests: Eurofit and quantification of physical activity through QAPACE questionnaire in a sample of 181 students ( 75 girls and 106 boys) aged between 9 and 12 years. The data obtained showed that the corresponding measurements to most physical tests show significant differences depending on gender, except for balance and flexibility.

Children showed better performance in physical tests than girls. In the amount of physical activity, children recorded higher values than girls with a tendency to decrease when they start growing up, whereas in girls the amount of physical activity is slightly constant in terms of increasing age.

Finally, to perform the Pearson correlation to a level of significance of $5 \%$ was found positive for most variables coefficients, but small (Balance $r=0.025$, explosive strength $r=0.063$, trunk strength $r=0.086$, isometric force $r=0.087$ and cardio respiratory endurance $r=0.013$, reflect a direct but weak relationship showing that children who practice greater amounts of physical activity show slightly better motor conditions in these areas.

Key words: Motor development, children, physical activity, production of results.

\footnotetext{
1 Magister en Pedagogía de la Cultura Física. Docente Universidad Pedagógica y Tecnológica de Colombia. Grupo de investigación FIMED. E-mail:fernandojavieravilacorrea@yahoo.es
} 


\section{Introducción}

Todas las funciones orgánicas en el ser humano tienen períodos de maduración, de optimización, de mantenimiento y luego de declinación. De acuerdo con Guzmán (2010), el desarrollo motriz en la infancia y niñez está relacionado con la maduración neuromuscular, el rápido crecimiento del sistema nervioso y diversos aspectos psicosociales. Una vez que los patrones básicos de movimiento están establecidos, el aprendizaje y la práctica son factores que influyen significativamente sobre la competencia motriz, además de las características de crecimiento y maduración del niño. Los beneficios de la actividad física a lo largo de la vida son indiscutibles, tanto en el plano físico como en el psicológico, social y moral; además, la participación de los niños y adolescentes en actividades físicas escolares y extraescolares, determinan y afectan su desarrollo motor y cognitivo, según se ha demostrado en estudios llevados a cabo dentro de contextos específicos y con diferentes grupos de población.

En Colombia los estudios sobre actividad física se realizan básicamente en términos exploratorios, como el de Robledo (2006), que asocia la actividad física con las características regionales; también hay estudios comparativos y analíticos que buscan establecer relaciones con aspectos como obesidad y frecuencia cardiaca basal (Giraldo et al., 2008; Londoño 2008; Melgarejo, 2011).

En este sentido, el presente estudio busca obtener información veraz y válida referente al desarrollo motriz y actividad física para el contexto educativo de los niños Chiquinquireños. Mediante la observación y la aplicación de instrumentos validados para la población seleccionada, se busca conocer los niveles de desarrollo motriz y actividad física en escolares de grado quinto primaria y la forma como estos se relacionan, a fin de ofrecer puntos de partida sólidos para futuras intervenciones y procesos investigativos.
Para el desarrollo motriz se utilizó la batería Eurofit test, propuesta por el Comité de expertos del Consejo de Europa, que aborda la evaluación de la aptitud física bajo características morfológicas, capacidad motora, la cual es base de este estudio, y capacidad fisiológica (Ureña, 1998); el test fue diseñado para edades entre 6 y 18 años, aunque también ha sido aplicado con éxito a grupos de edad superior (Gómez, 2002). Estos test, que se aplicaron a más de 50000 alumnos europeos, quedaron definitivos y validados en Mayo de 1986 (Renson, 1987). En Colombia esta batería se ha utilizado en rangos de edades de 8 a 16 años, en estudios relacionados con actividad física y sobrepeso, condición física y nutrición, y para la evaluación de la condición física (Londoño, 2008; Maestre, 2010; Villera, 2010). Para la cuantificación de la actividad física se utilizó el cuestionario QAPACE, validado internacionalmente y para la población Colombiana, dentro de los rangos de edad útiles para el presente estudio (Barbosa, 2010).

El Objetivo general fue el de encontrar la relación entre el desarrollo motriz y la actividad física en estudiantes de grado quinto del Técnico Industrial Julio Flórez, del municipio de Chiquinquirá. En primer lugar se midió el grado de desarrollo motriz en los estudiantes, luego se calculó la cantidad de actividad física que realizan los niños, a fin de analizar el comportamiento de su desarrollo motriz y de su actividad física; finalmente, se correlaciona el desarrollo motriz y la cantidad de actividad física en estos niños. En los siguientes apartados se explican las etapas de la investigación, los criterios en la selección de los sujetos estudiados, los materiales utilizados, así como el análisis de datos y hallazgos más relevantes.

\section{Materiales y Métodos}

La línea de investigación bajo la cual se desarrolló el estudio es Actividad Física, el paradigma de investigación adoptado fue el positivista, sin 
intervención. El diseño utilizado fue no experimental correlacional, que se ajusta a encontrar posibles explicaciones o relaciones entre variables involucradas en el fenómeno de estudio (Valenzuela y Flórez, 2012).

El estudio se dividió en tres fases: evaluación de la motricidad en niños de quinto primaria del Instituto Técnico Industrial Julio Flórez de Chiquinquirá, por medio de la batería EUROFIT; evaluación de la actividad física de los mismos niños, con la encuesta QAPACE y finalmente, el análisis y correlación de la información a través de un método estadístico.

La población está integrada por estudiantes matriculados de grado quinto primaria de ambos géneros, de la Institución Educativa Técnico Industrial Julio Flórez, con 223 estudiantes. Para la selección de la muestra se establece que es de tipo probabilístico; se utilizó muestreo simple, de modo que la selección de los estudiantes se realizó al azar a partir del listado general.

Para establecer el tamaño de la muestra se utilizó la proporción del $50 \%$, con un nivel de confianza del $95 \%$ y una precisión del $1 \%$, de acuerdo a la ecuación 1.

$$
n=\frac{N z_{a}{ }^{2} p q}{d(N-1)+z_{a}{ }^{2} p q}
$$

Donde Z=1.96 y N es la población; d por lo general representa la precisión para obtener la muestra, $1 \%$ en éste caso.

Al reemplazar en la ecuación 1 se obtiene una muestra de 181 estudiantes. Se utilizó el paquete estadístico SPSS para seleccionar los sujetos, a partir del listado general de estudiantes de grado quinto de la institución educativa ya mencionada; se obtuvo una muestra conformada por 75 niñas y 106 niños, entre 9 y 12 años de edad. Dentro de estos grupos figuran 3 niños y 3 niñas de 9 años, 44 niños y 25 niñas de 10 años, 51 niños y 36 niñas de
11 años y, 8 niños y 11 niñas de 12 años.

Las variables dependientes en el estudio son: cantidad de actividad física y nivel de desarrollo motriz. Las variables independientes son: género, edad, peso, talla, nivel socioeconómico, equilibrio corporal, velocidad miembro superior, flexibilidad, fuerza estática, fuerza explosiva, fuerza isométrica, velocidad de coordinación y resistencia cardio respiratoria.

\section{Resultados y Discusión}

Para determinar la correlación entre el desarrollo motriz y la cantidad de actividad física, se hace un análisis de las diferentes pruebas motrices versus la cantidad de actividad física encontrados en los estudiantes que forman parte del estudio. De acuerdo con las fases definidas, los resultados obtenidos son:

En la evaluación de la motricidad en niños de quinto primaria del Instituto Técnico Industrial Julio Flórez de Chiquinquirá, por medio de la batería EUROFIT, los valores promedio encontrados en las diferentes pruebas físicas muestran mejores desempeños para los niños que para las niñas en la mayoría de variables motrices, como se observa en la tabla 1.

\begin{tabular}{lcc}
\hline \multirow{2}{*}{ Variable motriz } & \multicolumn{2}{c}{ Género } \\
\cline { 2 - 3 } & Masculino & Femenino \\
\cline { 2 - 3 } & Valor promedio & Valor promedio \\
\hline Equilibrio & 11 & 11 \\
Velocidad miembro superior & 153 & 166 \\
Flexibilidad & 11.7 & 11 \\
Fuerza explosiva & 113.1 & 99.5 \\
Fuerza estática & 18.6 & 16.1 \\
Fuerza del tronco & 22 & 18 \\
Fuerza isométrica & 6.6 & 3.2 \\
Velocidad de coordinación & 24.6 & 27.3 \\
Resistencia Cardiorespiratoria & 4 & 3 \\
\hline
\end{tabular}

Tabla 1. Promedios para las variables motrices 
Se realizó la prueba de normalidad para estas variables y se evidenció que se distribuyen de forma normal, ya que con un nivel de significancia del 5\% la prueba K-S así lo indica. En cuanto a la variación en las pruebas físicas, de acuerdo con la edad de los niños y niñas estudiados, se observó que el rendimiento en todas las pruebas fue mayor para los varones y aumenta directamente con la edad, ver tablas 2 y 3 .

\begin{tabular}{|c|c|c|c|c|c|c|c|c|c|}
\hline Edad & $\begin{array}{l}\text { Estadística } \\
\text { Descriptiva }\end{array}$ & $\begin{array}{l}\text { Velocidad de } \\
\text { extremidades } \\
\text { superiores }\end{array}$ & Flexibilidad & $\begin{array}{c}\text { Fuerza } \\
\text { explosiva }\end{array}$ & $\begin{array}{c}\text { Fuerza } \\
\text { estática }\end{array}$ & $\begin{array}{c}\text { Fuerza del } \\
\text { tronco }\end{array}$ & $\begin{array}{c}\text { Fuerza } \\
\text { isométrica }\end{array}$ & Velocidad & Resistencia \\
\hline \multirow{6}{*}{9} & $\begin{array}{l}\text { Percentil } \\
\text { promedio }\end{array}$ & 50.0 & 60.0 & 5.0 & 20.0 & 95.0 & 15.0 & 5.0 & 20.0 \\
\hline & $\begin{array}{l}\text { Desviación } \\
\text { estándar }\end{array}$ & 30.6 & 30.1 & 7.2 & 28.4 & 2.3 & 7.6 & 2.9 & 8.7 \\
\hline & Valor Máximo & 90.0 & 85.0 & 15.0 & 60.0 & 99.0 & 20.0 & 10.0 & 20.0 \\
\hline & Valor mínimo & 30.0 & 25.0 & 1.0 & 5.0 & 95.0 & 5.0 & 5.0 & 5.0 \\
\hline & Mediana & 50.0 & 60.0 & 5.0 & 20.0 & 95.0 & 15.0 & 5.0 & 20.0 \\
\hline & Moda & NA & NA & NA & NA & 95,0 & NA & 5.0 & 20.0 \\
\hline \multirow{6}{*}{10} & $\begin{array}{l}\text { Percentil } \\
\text { promedio }\end{array}$ & 30.0 & 10.0 & 5.0 & 40.0 & 95.0 & 15.0 & 5.0 & 15.0 \\
\hline & $\begin{array}{l}\text { Desviación } \\
\text { estándar }\end{array}$ & 26.4 & 17.6 & 13.0 & 33.8 & 26.4 & 23.7 & 6.5 & 22.8 \\
\hline & Valor Máximo & 95.0 & 70.0 & 45.0 & 99.0 & 99.0 & 85.0 & 25.0 & 75.0 \\
\hline & Valor mínimo & 1.0 & 1.0 & 1.0 & 1.0 & 1.0 & 1.0 & 1.0 & 1.0 \\
\hline & Mediana & 42.5 & 10.0 & 5.0 & 30.0 & 80.0 & 12.5 & 5.0 & 15.0 \\
\hline & Moda & 10.0 & 5.0 & 1.0 & 30.0 & 90.0 & 5.0 & 1.0 & 5.0 \\
\hline \multirow{6}{*}{11} & $\begin{array}{l}\text { Percentil } \\
\text { promedio }\end{array}$ & 30.0 & 20.0 & 10.0 & 15.0 & 80.0 & 25.0 & 10.0 & 20.0 \\
\hline & $\begin{array}{l}\text { Desviación } \\
\text { estándar }\end{array}$ & 21.4 & 19.1 & 22.2 & 25.9 & 21.5 & 23.4 & 12.5 & 22.5 \\
\hline & Valor Máximo & 80.0 & 80.0 & 80.0 & 95.0 & 99.0 & 90.0 & 55.0 & 90.0 \\
\hline & Valor mínimo & 1.0 & 1.0 & 1.0 & 1.0 & 15.0 & 5.0 & 1.0 & 1.0 \\
\hline & Mediana & 35.0 & 20.0 & 5.0 & 25.0 & 85.0 & 25.0 & 5.0 & 15.0 \\
\hline & Moda & 35.0 & 5.0 & 1.0 & 1.0 & 85.0 & 5.0 & 5.0 & 30.0 \\
\hline \multirow[t]{6}{*}{12} & $\begin{array}{l}\text { Percentil } \\
\text { promedio }\end{array}$ & 30.0 & 1.0 & 1.0 & 15.0 & 75.0 & 25.0 & 5.0 & 5.0 \\
\hline & $\begin{array}{l}\text { Desviación } \\
\text { estándar }\end{array}$ & 12.8 & 3.3 & 4.2 & 31.1 & 14.4 & 27.6 & 9.8 & 13.2 \\
\hline & Valor Máximo & 45.0 & 10.0 & 10.0 & 75.0 & 95.0 & 85.0 & 20.0 & 30.0 \\
\hline & Valor mínimo & 5.0 & 1.0 & 1.0 & 1.0 & 45.0 & 15.0 & 1.0 & 1.0 \\
\hline & Mediana & 30.0 & 1.0 & 1.0 & 3.5 & 70.0 & 27.5 & 1.0 & 1.0 \\
\hline & Moda & 35.0 & 1.0 & 1.0 & 1.0 & 70.0 & 20.0 & 1.0 & 1.0 \\
\hline
\end{tabular}

Tabla 2. Estadística descriptiva para percentiles de pruebas físicas en los niños clasificados por edad

Durante la fase de evaluación de la actividad física en los niños estudiados con la encuesta QAPACE, se obtuvieron los datos que se muestran en la tabla 4.
Los resultados muestran mayores niveles de actividad física en los niños que en las niñas, con una tendencia en los niños a disminuir los niveles de actividad a medida que aumenta la edad 


\begin{tabular}{|c|c|c|c|c|c|c|c|c|c|}
\hline Edad & $\begin{array}{l}\text { Estadística } \\
\text { Descriptiva }\end{array}$ & $\begin{array}{l}\text { Velocidad de } \\
\text { extremidades } \\
\text { superiores }\end{array}$ & Flexibilidad & $\begin{array}{l}\text { Fuerza } \\
\text { explosiva }\end{array}$ & $\begin{array}{l}\text { Fuerza } \\
\text { estática }\end{array}$ & $\begin{array}{l}\text { Fuerza } \\
\text { del } \\
\text { tronco } \\
\end{array}$ & $\begin{array}{l}\text { Fuerza } \\
\text { isométrica }\end{array}$ & Velocidad & Resistencia \\
\hline & $\begin{array}{l}\text { Percentil } \\
\text { promedio }\end{array}$ & 35.0 & 40.0 & 10.0 & 10.0 & 95.0 & 40.0 & 10.0 & 15.0 \\
\hline \multirow[t]{6}{*}{9} & $\begin{array}{l}\text { Desviación } \\
\text { estándar }\end{array}$ & 8.7 & 11.5 & 2.9 & 7.6 & 5.8 & 34.0 & 2.9 & 2.9 \\
\hline & Valor Máximo & 45.0 & 55.0 & 10.0 & 20.0 & 95.0 & 85.0 & 10.0 & 20.0 \\
\hline & Valor mínimo & 30.0 & 35.0 & 5.0 & 5.0 & 85.0 & 20.0 & 5.0 & 15.0 \\
\hline & Mediana & 30.0 & 35.0 & 10.0 & 15.0 & 95.0 & 35.0 & 10.0 & 20.0 \\
\hline & Moda & 30.0 & 35.0 & 10.0 & NA & 95.0 & NA & 10.0 & 20.0 \\
\hline & $\begin{array}{l}\text { Percentil } \\
\text { promedio }\end{array}$ & 10.0 & 5.0 & 5.0 & 80.0 & 70.0 & 25.0 & 1.0 & 20.0 \\
\hline \multirow[t]{6}{*}{10} & $\begin{array}{l}\text { Desviación } \\
\text { estándar }\end{array}$ & 15.3 & 16.3 & 24.1 & 29.9 & 24.2 & 17.5 & 7.7 & 24.2 \\
\hline & Valor Máximo & 65.0 & 75.0 & 95.0 & 95.0 & 96.0 & 75.0 & 25.0 & 85.0 \\
\hline & Valor mínimo & 1.0 & 1.0 & 1.0 & 1.0 & 10.0 & 15.0 & 1.0 & 5.0 \\
\hline & Mediana & 10.0 & 5.0 & 1.0 & 60.0 & 75.0 & 25.0 & 1.0 & 15.0 \\
\hline & Moda & 5.0 & 1.0 & 1.0 & 95.0 & 80.0 & 20.0 & 1.0 & 5.0 \\
\hline & $\begin{array}{l}\text { Percentil } \\
\text { promedio }\end{array}$ & 10.0 & 5.0 & 5.0 & 25.0 & 65.0 & 25.0 & 1.0 & 10.0 \\
\hline \multirow[t]{6}{*}{11} & $\begin{array}{l}\text { Desviación } \\
\text { estándar }\end{array}$ & 19,3 & 10.0 & 10,4 & 23,6 & 22,7 & 19,4 & 5,2 & 21,2 \\
\hline & Valor Máximo & 80.0 & 40.0 & 55.0 & 80.0 & 99.0 & 65.0 & 25.0 & 75.0 \\
\hline & Valor mínimo & 1.0 & 1.0 & 1.0 & 1.0 & 1.0 & 5.0 & 1.0 & 1.0 \\
\hline & Mediana & 10.0 & 1.0 & 1.0 & 30.0 & 65.0 & 20.0 & 1.0 & 10.0 \\
\hline & Moda & 5.0 & 1.0 & 1.0 & 30.0 & 40.0 & 10.0 & 1.0 & 1.0 \\
\hline & $\begin{array}{l}\text { Percentil } \\
\text { promedio }\end{array}$ & 5.0 & 1.0 & 1.0 & 25.0 & 20.0 & 25.0 & 1.0 & 10.0 \\
\hline \multirow[t]{5}{*}{12} & $\begin{array}{l}\text { Desviación } \\
\text { estándar }\end{array}$ & 14.6 & 4.3 & 0.0 & 19.3 & 21.2 & 15.5 & 3.6 & 14.5 \\
\hline & Valor Máximo & 45.0 & 15.0 & 1.0 & 80.0 & 75.0 & 50.0 & 10.0 & 50.0 \\
\hline & Valor mínimo & 1.0 & 1.0 & 1.0 & 15.0 & 10.0 & 10.0 & 1.0 & 1.0 \\
\hline & Mediana & 5.0 & 1.0 & 1.0 & 30.0 & 20.0 & 25.0 & 1.0 & 5.0 \\
\hline & Moda & 1.0 & 1.0 & 1.0 & 30.0 & 15.0 & 25.0 & 1.0 & 5.0 \\
\hline
\end{tabular}

Tabla 3. Estadística descriptiva para percentiles de pruebas físicas en las niñas clasificados por edad

\begin{tabular}{|c|c|c|c|}
\hline \multirow{2}{*}{\multicolumn{2}{|c|}{ Genero }} & \multicolumn{2}{|c|}{ Promedio de gasto energético (Kjl/ día) } \\
\hline & & Masculino & Femenino \\
\hline \multirow{4}{*}{ Edad (años) } & 9 & 217.43 & 157.29 \\
\hline & 10 & 173.00 & 159.98 \\
\hline & 11 & 170.01 & 156.24 \\
\hline & 12 & 168.18 & 160.49 \\
\hline
\end{tabular}

Tabla 4. Cantidad promedio de actividad física por género y edad

(relación inversa y débil); en las niñas, aunque los niveles de actividad física son menores, se mantienen con poca variación a medida que aumenta la edad.
Durante la última fase, análisis y correlación por medio de un método estadístico, se procede a realizar la correlación de Pearson a un nivel de significancia del $5 \%$ obteniendo los datos que se muestran en la tabla 5. 


\begin{tabular}{lc}
\hline Variable Motriz (Correlación de Pearson $(\mathbf{r})$ ) & Gasto Enerqético: KJI \\
\hline Equilibrio & 0.025 \\
Velocidad miembro superior & -0.056 \\
Flexibilidad & -0.014 \\
Fuerza explosiva & 0.063 \\
Fuerza estática & -0.039 \\
Fuerza del tronco & 0.086 \\
Fuerza isométrica & 0.087 \\
Velocidad de coordinación. & -0.068 \\
Resistencia Cardiorespiratoria & 0.013 \\
\hline
\end{tabular}

Tabla 5. Correlación de pearson actividad física y variables motrices

Luego de revisar los análisis físico, motriz y los coeficientes de Pearson para los distintos grupos de variables se encuentra que para la mayoría los coeficientes son positivos, aunque pequeños: equilibrio $r=0.025$, fuerza explosiva $r=0.063$, fuerza del tronco $r=0.086$, fuerza isométrica $r=0.087 \mathrm{y}$ resistencia cardio respiratoria $r=0.013$; los valores anteriores reflejan una relación directa pero débil.

Los resultados también muestran que el rendimiento en todas las pruebas fue mayor para los varones y aumenta directamente con la edad. Cuando se toma en cuenta el nivel de actividad física se observa que para los estudiantes que tienen mayores niveles, el desarrollo motriz y por consiguiente su desempeño en las diferentes pruebas es notablemente mejor, que en quienes tienen niveles de actividad física bajos.

Se encontraron diferencias significativas teniendo en cuenta el género en todas las medidas, los niños superaron a las niñas en todas las pruebas excepto en las de equilibrio y flexibilidad. Los datos se dividieron en tercios, en función del nivel de actividad física, teniendo que entre más alto nivel de actividad física mayor aptitud anaeróbica, mayor resistencia cardiorespiratoria y mayores niveles de fuerza; mientras que los grupos de bajos niveles de actividad física, aunque muestran menor capacidad anaeróbica y cardiorespiratoria, tienen una mayor flexibilidad y equilibrio.

En estudios como el de Vedul-Kjelsås et al. (2012), de tipo correlacional, se halló una correlación alta y significativa, 0.71, entre la aptitud física y el desarrollo motriz para ambos sexos, a diferencia del presente estudio en el que se observa, para las variables motrices y la actividad física, una correlación débil. La diferencia puede obedecer a las condiciones antropométricas de los sujetos estudiados en ambos casos: por una parte, VedulKjelsås et al. (2012) trabajaron con 67 niños, con edad promedio de 11.46 años, cuyas condiciones de peso y talla figuraban dentro de los rangos normales, mientras que en el presente estudio, la mayoría de los niños y niñas presentan condiciones de delgadez por debajo de los índices normales.

Ericsson (2011), estudió los efectos sobre el desarrollo motriz de un programa de intervención en actividad física con un grupo de niños de 9 y 11 años. En su diagnóstico inicial encontró una correlación débil entre el desarrollo motriz de los niños y la cantidad de actividad física, $r<0.2$ además encontró marcadas diferencias en la condición motriz entre niños y niñas. Luego de su intervención, las habilidades motoras de los estudiantes mejoraron, las diferencias de género se mostraron disminuidas y la significancia de la correlación tuvo un incremento $r=0.38$ pero se mantuvo baja. Estos datos comparados con los obtenidos en el presente estudio son similares, ya que los coeficientes encontrados entre las variables motrices y la actividad física son pequeños $r<0.18$, y también se notan diferencias de género en cuanto al desempeño motriz; en este caso los niños muestran mejores resultados en las pruebas físicas que las niñas y mayores niveles de actividad física.

Lopes et al. (2011), buscaron la correlación entre 
actividad física y competencia motriz, encontrando una tendencia general a la disminución en el nivel de actividad física con el incremento en la edad, dependiendo del nivel inicial de competencia motriz. La tasa estimada de disminución de la actividad física fue insignificante para los niños con mayores niveles de desarrollo motriz a los 6 años, pero se amplía con 2.58 y 2.47 unidades cada año, respectivamente, para los niños con niveles bajos y medios de competencia inicial. Estos resultados coinciden en gran medida con lo encontrado en los niños del presente estudio, ya que también se tiene la tendencia a disminuir los niveles de actividad física con el aumento de la edad, comportamiento más marcado en los niños que en las niñas.

Castelli et al. (2007), en su estudio correlacional también encontraron diferencias significativas de género en todas las medidas, excepto en la competencia motora. Los niños superaron a las niñas en todas las pruebas físicas excepto en flexibilidad. Las correlaciones establecidas para muchas de las variables analizadas estuvieron entre moderada y débil tanto en hombres como en mujeres. La relación entre actividad física y competencia motora fue débil, pero positiva, tanto para los hombres con $r=0.16$, como para las mujeres, con $r=0.23$. Nuevamente se presentan resultados estadísticamente similares con las mediciones realizadas en el presente estudio, diferencias marcadas en las medidas dependiendo del género y relación débil pero directa $r<0.18$.

Haga (2009), analizó una muestra de 67 niños con edad media de 9.7 años, para evaluar la competencia motora; los resultados evidencian una fuerte y significativa relación positiva entre la competencia motriz y la aptitud física asociada a niveles altos de actividad física, $r=0.78$; en éste caso, aunque el coeficiente de correlación difiere en magnitud del encontrado en los niños del Técnico Industrial Julio Flórez, su comportamiento indica también una relación directa.
Blaes et al. (2011), trabajaron con 86 niños y niñas franceses de 6 a 12 años, a fin de encontrar la relación entre el rendimiento motriz (test Eurofit) y la actividad física. En su trabajo no se encontró relación entre la actividad física y el rendimiento físico. En los varones se observó que la grasa corporal se asoció negativamente con la actividad física vigorosa, $r=-0.38$ y $p<0.001$, y la actividad física muyalta, $r=-0.35$ y $p<0.01$,en contraste con la actividad física baja $(r=0.28, p<0.01)$, que se relaciona positivamente con la cantidad de grasa corporal.

Wrotniak et al. (2006), realizaron un estudio de tipo correlacional que incluyó 34 niñas y 31 niños, en los que la competencia motriz se asoció positivamente con la actividad física, $r=0.46$, e inversamente con la actividad sedentaria en los niños, $r=0.39$. Comparando estos resultados con los propios del presente estudio se evidencia que en ambos casos se presenta una relación positiva entre las dos variables aunque con significancias en el primer caso media y en el segundo débil, pero conservando equivalencias de comportamiento estadístico.

\section{Conclusiones}

El análisis del desarrollo motriz de los niños de grado quinto del Técnico Industrial Julio Flórez, del municipio de Chiquinquirá, muestra que las mediciones correspondientes a la mayoría de las pruebas físicas presentan diferencias significativas e importantes dependiendo del género, excepto para equilibrio y flexibilidad. Los niños muestran mejores desempeños en las pruebas físicas que las niñas. Además, la cantidad de actividad física es mayor en los niños que en las niñas; en ellos se nota una tendencia a la disminución de estas cantidades a medida que aumenta la edad, mientras que las niñas presentan valores de actividad física ligeramente constantes en términos del aumento de la edad. La relación entre el desarrollo motriz y la cantidad de actividad física en los niños estudiados es directa pero débil. 
Debido a la correlación directa que presentan la actividad física y el desarrollo motriz de los niños y las niñas en edad escolar, es importante que el currículo de Educación Física en la básica primaria sea constantemente revisado y ajustado a las necesidades de los estudiantes; de ésta manera se podrán incluir estrategias para el fomento de la práctica regular y planificada de diversas actividades físicas dentro y fuera de las escuelas, que permitan el mejoramiento de las habilidades y capacidades motrices básicas y específicas.

La revisión documental muestra la necesidad de llevar a cabo procesos investigativos de validación de instrumentos para la evaluación del desarrollo motor en los niños en edad escolar. Los estudios de evaluación del desarrollo motor y la cuantificación de la actividad física permiten al educador físico informarse adecuadamente de los avances y logros de los estudiantes en materia de movimiento; de ésta forma será posible que efectúen las correcciones o intervenciones a que haya lugar.

\section{Referencias}

Barbosa, N., Sánchez C., Vera, J., Perez, W., Thalabard J., \& Rieu. M. (2007). A physical activity questionnaire: Reproducibility and validity. Journal of Sports Science and Medicine, 6, 505518.

Blaes, A., Baquet, G., Fabre, C., Van P., \& Berthoin, S. (2011). Is there any relationship between physical activity level and patterns, and physical performance in children? International Journal of Behavioral Nutrition and Physical Activity, 8(1), 122.

Castelli, D., \& Valley, J. (2007). Chapter 3: The Relationship of Physical Fitness and Motor Competence to Physical Activity. Journal of Teaching in Physical Education, 26(4), 358-374.

Ericsson, I. (2011). Effects of Increased Physical Activity on Motor Skills and Marks in Physical
Education: An Intervention Study in School Years 1 through 9 in Sweden. Physical Education and Sport Pedagogy, 16(3), 313-329.

Giraldo, D., Poveda, E., Forero, Y., Mendivil, C., \& Castro, L. (2008). Actividad física autorreportada, comparación con indicadores antropométricos de grasa corporal en un grupo de escolares de Bogotá y de cinco departamentos del centro oriente de Colombia 2000-2002. Biomédica, 28, 386-395.

Gómez, J., Berral de la Rosa, C.J., Viana, B., \& Berral de la Rosa, F.J. (2002). Valoración de la aptitud física en escolares. Archivos de medicina del deporte, 19(90), 273-282.

Guzmán, R. (2010). Valoración Médico Deportiva: Aspectos biopsicosociales relacionados con las actividades físicas y deportivas en niños y adolescentes. Rev Clin Med Fam [online], 3(3), 192 200. DOI: http://dx.doi.org/10.4321/S1699695X2010000300007.

Haga, M. (2009). Physical fitness in children with high motor competence is different from that in children with low motor competence. Phys Ther, 89(10), 1089-97.

Lopes, V., Rodrigues, L., Maia, J., \& Malina, R. (2011). Motor coordination as predictor of physical activity in childhood. Scand J Med Sci Sports, 21(5), 663-9.

Londoño, C., Barbosa, N., Tovar, G., \& Sánchez, C. (2009). Sobrepeso en escolares: prevalencia, factores protectores y de riesgo en Bogotá. Bogotá: Universidad del Rosario.

Maestre, J., \& Cela, C. (2010). Relación entre el estado nutricional y la condición física en población en edad escolar. Journal of Sport and Health Research, 2(2), 95-108.

Melgarejo, V., Barbosa, N., Patiño, E., \& Salcedo, L. (2011). Cuantificación de la actividad física y comportamiento de la frecuencia cardiaca basal, 
en reposo y máxima, en escolares de 8 a 16 años, en altura. Rev.salud.hist.sanid, on-line 6(2). Recuperado de: file:///C:/Users/user/Downloads/ 1908-2135-1-PB.pdf.

Renson, R. (1987). Sélection et principles de base des tests d'évaluation de l'aptitude motrice "Eurofit", en CDDDS. V Séminaire Européen de Recherche sur l'evaluation de l'aptitude physique. Scoula Nazionale d'Atletica Leggera, Formia (Italie), 12-17 mai 1986. Comité pour le développment du sport. Strasbourg. 88-113.

Robledo-Martinez, R. (2006). Características Socioculturales de la Actividad Física en Tres Regiones de Colombia. Rev.Salud pública, 8(2), 1327.

Vedul-Kjelsås, V., Sigmundsson, H., Stensdotter, A., \&Haga, M. (2012). The relationship between motor competence, physical fitness and self-perception in children. Child Care Health Dev, 38(3), 394-402.
Ureña, A. (1998). Incidencia de la función ofensiva sobre el rendimiento de la recepción del saque en voleibol. (Tesis doctoral, Departamento de Personalidad, Evaluación y Tratamiento Psicológico). Universidad de Granada.

Valenzuela, J., \& Flórez, M. (2012). Fundamentos de Investigación Educativa. México: Tecnológico de Monterrey.

Villera, S., \& Petro, J. (2010). Valoración de la aptitud física de los escolares de 10 a 12 años de Montería, Colombia. EFDeportes.com, Revista digital. Buenos aires, 15(148). Recuperado de: http://www.efdeportes.com/efd148/valoracionde-la-aptitud-fisica-de-los-escolares.htm.

Wrotniak, B., Epstein, L., Dorn, J., Jones, K., \& Kondilis, V. (2006). The Relationship Between Motor Proficiency and Physical Activity in Children. Pediatrics, 118(6), e1758-e1765. 